\title{
Das Differenzierungspotential eines mehrdimensionalen Kriterienkatalogs zur Bewertung von Onlineshops: Eine empirische Untersuchung in einem Schweizer Portal
}

\author{
Achim Dannecker, Michael Quade \\ Competence Center E-Business Basel, \\ Fachbochschule Nordwestschweiz FHNW
}

\section{Einleitung}

Im Jahr 2006 sagte Tim O'Reilly auf dem Dresdner Zukunftsforum voraus: „In ein paar Jahren werden die Prinzipien des Web 2.0 absolut Mainstream sein“. Er empfahl demzufolge: „Sie müssen die kollektive Intelligenz der User nutzbar machen“ (Hage 2006). Heute sind viele der Prinzipien des Web 2.0 allgegenwärtig, darunter Bewertungen von Inhalten im Internet.

Internetdienste, die dem Nutzer Bewertungen zu Inhalten ermöglichen und anbieten, können sich einen Vorteil gegenüber Wettbewerbern schaffen.

Die eigentliche Herausforderung besteht in der Definition des Umfangs eines Kriterienkatalogs. Zum einen soll die Bewertung in ihrer Ausprägung dem Nutzer einen einfachen (quasi auf ein Kriterium reduzierten) Eindruck geben, um ihn bei der Transaktionsentscheidung zu unterstützen. Zum anderen soll der Nutzer aufgrund einer möglichst differenzierten Bewertung entscheiden können. Für den Nutzer muss es so einfach wie möglich sein, eine Bewertung abzugeben - und es soll ihm Spaß machen (van der Heijden 2004, S. 1). Die Bewertung soll aber auch so umfangreich sein, dass andere Nutzer ein präzises Bild vom bewerteten Produkte erhalten.

In dieser Arbeit wird ein Portal untersucht, das Onlineshops in der Schweiz auflistet, und diese durch Nutzer des Portals bewerten lässt. Aus dem methodischen Vorgehen der Analyse wird darüber hinaus eine Vorgehensweise entwickelt, die aufzeigt, wie die Qualität (Redundanz vs. Differenzierung) eines Kriterienkatalogs mittels einer explorativen Faktorenanalyse untersucht werden kann.

Diese Arbeit ist wie folgt aufgebaut: Im zweiten Kapitel wird der aktuelle Stand der Forschung aufgezeigt. Es wird dargestellt, in welchem Kontext und mit welchem Nutzen Bewertungen ermöglicht und angeboten werden. Weiter werden Seiten im Internet aufgelistet, die Bewertungsfunktionalitäten in unterschiedlichen 
Bereichen anbieten. Im dritten Kapitel wird das methodische Vorgehen bei der Extraktion der Daten, deren Aufbereitung für die anschließenden explorativen Faktorenanalyse aufgezeigt. Im vierten Kapitel werden die Ergebnisse der explorativen Faktorenanalyse besprochen. Den Abschluss bildet eine Diskussion über Handlungsoptionen für zukünftige Entwicklungen von Bewertungsplattformen.

\section{Aktueller Stand der Forschung}

Die traditionelle Geschäftsbeziehung basiert auf Vertrauen, das sich über eine gewisse Zeit hinweg auf der Basis persönlicher Kontakte aufgebaut hat. In den Situationen, wo eine Interaktion zwischen zwei Parteien stattfindet, gründet diese fundamental auf Reputation (Shmatikov und Talcott 2005, S. 188). Auf einer Plattform im Internet ist dies anders. Käufer und Verkäufer können sich synchron oder asynchron im Internet finden und brauchen sich nicht zu kennen. Die Beziehung bleibt anonym und auf eine bestimmte Transaktion im Internet beschränkt. Dies bedeutet, dass sich eine persönliche Beziehung in der Kürze nicht aufbauen kann und somit auch kein wechselseitiges Vertrauen aufgebaut wird. Dieses Vertrauen ist aber notwendig, um eine erfolgreiche Transaktion zwischen den Transaktionspartnern stattfinden zu lassen. Reputationssysteme können hierbei unterstützend wirken, indem sie eine Mediatorrolle übernehmen und auf diese Weise Vertrauen zwischen den Transaktionspartner aufbauen (Resnick et al. 2000, S. 46; Riemer und Korn 2007, S. 866). Die Aufgabe eines Reputationssystems ist es, die schon vorhandenen Bewertungen zu verwenden, um eine glaubwürdige Reputation eines Transaktionspartners zu berechnen. Die so berechnete Reputation soll der jeweils anderen Transaktionsseite einen Einblick geben, wie sich der Transaktionspartner voraussichtlich in der Zukunft verhalten wird und wie dessen Servicequalität sein wird (Dellarocas 2000, S. 1). Ein Problem, das hierbei entstehen kann, sind Bewertungen, die zu Unrecht negativ sind. Die Kontrolle der Anonymität kann unterstützend wirken, um solche Seiteneffekte zu vermeiden. Kontrolle bedeutet hierbei, dass der jeweils andere Transaktionspartner beispielsweise nur einen sogenannten „Nickname“ erfährt, sich aber sicher sein kann, dass der Transaktionspartner beim Betreiber der Plattform bekannt ist.

Bewertungen reflektieren ein Verhaltensmuster aus der Vergangenheit. Sie geben ass negative Bewertungen dabei wesentlich höher gewichtet werden als positive, zeigt das Beispiel eBay (Bryan et al. 2000). Durch eine positive Reputation kann eine Geschäftsbeziehung sehr positiv beeinflusst werden, dennoch kann sie nicht alleinig darauf aufgebaut werden (Friedman und Resnick 1999, S. 3). Die Reputation entwickelt sich mit der Anzahl der Bewertungen. Je mehr Bewertungen einer Transaktionsseite zugeordnet sind, desto stärker manifestiert sich die Reputation. In den meisten Fällen beginnt der Effekt der Reputation bereits bei den ersten Bewertungen und ist in der Startphase am stärksten. In dieser Phase bemühen sich 
Verkäufer wie auch Käufer besonders, eine gute Reputation zu erlangen (Dellarocas 2003, S. 1415).

Die Reputation eines Verkäufers kann auch als ein Indikator seiner Glaubwürdigkeit angesehen werden (Melnik und Alm 2002, S. 347-348; Shmatikov und Talcott 2005, S. 167). Sofern Handel auf der Basis eines Reputationssystems betrieben wird, finden nicht nur die Transaktionspartner, sondern auch das Reputationssystem selbst positive Beachtung. Dieser positive Effekt wird weitere Teilnehmer anziehen, die einen Beitrag zum Bewertungssystem leisten wollen. Das fördert den Wettbewerb zwischen den Verkäufern, was wiederum die Anstrengungen der Verkäufer erhöhen wird, gute Bewertungen zu bekommen. (Kollock 1999). Cabral und Hortaçsu haben nachgewiesen, dass es einen signifikanten Zusammenhang zwischen den Verkaufszahlen eines Verkäufers und der Reputation des Verkäufers auf dem System gibt (Cabral und Hortaçsu 2006, S. 33-34). In Tabelle 1 wird eine exemplarische Auflistung verschiedener Bewertungsplattformen aufgezeigt, um die unterschiedlichen Ausprägungen von Kriterienkatalogen und Bewertungsinhalten darzustellen.

\section{Tabelle 1: Auflistung Portale mit Bewertungen}

\begin{tabular}{|l|l|l|}
\hline Website & $\begin{array}{l}\text { Bewertungs- } \\
\text { gegenstand }\end{array}$ & Bewertungssystematik \\
\hline hrs.de & $\begin{array}{l}\text { Hotels } \\
\text { Bewertungen }\end{array}$ & 13 Kriterien \\
\hline holidaycheck.de & $\begin{array}{l}\text { Urlaubsreisen } \\
\text { Bewertungen }\end{array}$ & Anzahl Kriterien variiert $(>6)$ \\
\hline ciao.de & $\begin{array}{l}\text { Produkte } \\
\text { Bewertungen }\end{array}$ & Anzahl Kriterien variiert $(>6)$ \\
\hline MeinProf.de/ch & Personen & 7 Kriterien \\
\hline toppreise.ch & Onlineshops & 12 Kriterien \\
\hline Preisvergleich.ch & Onlineshops & 4 Kriterien \\
\hline eBay.de/ch & $\begin{array}{l}\text { Transaktions- } \\
\text { partner }\end{array}$ & $\begin{array}{l}\text { Verkäufer } 4 \text { Kriterien } \\
\text { Käufer 1 Kriterium }\end{array}$ \\
\hline amazon.de & $\begin{array}{l}\text { Produkte } \\
\text { Rezensionen }\end{array}$ & 1 Kriterium \\
\hline guenstiger.de & Produkte & 1 Kriterium \\
\hline
\end{tabular}

Es gibt eine ganze Reihe unterschiedlicher Portale mit jeweils unterschiedlich ausgeprägter Implementierung der Bewertungsfunktionalität bzw. des Kriterienkatalogs. Vor allem in der Anzahl der Kriterien variieren die Portale. Für den von uns gewählten Ansatz der Faktoranalyse eignen sich nur Bewertungsgegenstände, die durch mehr als sechs Kriterien bewertet werden, um wenigstens drei Variablen je Faktor zu gewährleisten. Die Literatur zeigt hierfür keine Vorgehensweise auf, wie bei der Auswahl des Kriterienkatalogs vorzugehen ist. Im Folgenden wird an einem konkreten Beispiel untersucht, inwiefern ein Kriterienkatalog geeignet ist, Inhalte zu differenzieren. 


\section{Methodisches Vorgehen}

Die Daten, für den in Kapitel 4 mittels der Faktorenanalyse beispielhaft untersuchten Kriterienkatalog, wurden im Rahmen einer Studie der Fachhochschule Nordwestschweiz erhoben. Die Studie hat zum Ziel, Schweizer Onlineshops auf Eigenschaften wie z.B. Produktpräsentation (Darstellung und Information), Möglichkeiten für die Suche nach Produkten, Warenkorbfunktionalität und Checkout-Prozess und Web-2.0-Funktionen wie Bewertungen und Foren hin zu untersuchen. Als Ausgangspunkt für die Studie wurden Preisvergleichsportale gewählt, da diese bereits Informationen über die oben genannten Eigenschaften der Onlineshops anbieten.

Die folgenden beiden Kapitel beschreiben wie die Preisvergleichsportale als mögliche Datenquellen evaluiert und ausgewählt und wie die Daten aus der gewählten Quelle extrahiert und aufbereitet wurden.

\subsection{Evaluation und Auswahl möglicher Datenquellen}

Im Ganzen wurden vier Schweizer Preisvergleichsportale als mögliche Informationsquelle, zu den Eigenschaften von Onlineshops, evaluiert (Anzahl Onlineshops gemäß Zugriff am 2. August 2009):

- comparis.ch (ca. 150 verzeichnete Onlineshops)

- guenstiger.ch (Anzahl verzeichneter Onlineshops unklar)

- preisvergleich.ch (Anzahl verzeichneter Onlineshops unklar)

- toppreise.ch (342 verzeichnete Onlineshops)

Die Portale bieten unterschiedlich viel Information über die Onlineshops an. Je nach Portal werden Informationen über die möglichen Zahlungsverfahren, die Versandoptionen, die Kontaktmöglichkeiten mit dem Anbieter und die Funktionalität des Shops (z.B. Warenkorb, Suchfunktion) angeboten.

Außer bei günstiger.ch wird bei allen Portalen eine Bewertung der Onlineshops nach mehreren Kriterien angegeben. Die Bewertung bei toppreise.ch besteht aus 12 Kriterien, die in 2 Blöcke aufgeteilt sind (vgl. Abbildung 1). Bei preisvergleich.ch können vier Kriterien bewertet werden: Präsentation und Navigation, Information und Service, Bestellung und Kosten, Lieferung. Bei comparis.ch sind sechs Bewertungskriterien angegeben: Zahlungsarten, Versand, Bestellung, Konsumentenrecht, Kontaktkanäle, Informationen auf der Webseite. Da comparis.ch die Onlineshops nicht durch Kunden bewertet lässt, sondern die Bewertung selbst vorgenommen hat ${ }^{1}$, wurde dieses Portal nicht weiter in die Untersuchung mit eibezogen.

toppreise.ch wurde als Datenquelle für die Studie ausgewählt, denn das Portal bietet allen oben genannten Informationen zu einem Onlineshop an. Ebenso sind

\footnotetext{
${ }^{1}$ www.comparis.ch/pricefinder/info/glossar.aspx?id=PF_Info_Hinweis_Berechnung_ComparisPoin ts Zugriff am 1. August 2009
} 
bei toppreise.ch im Vergleich zu den anderen Portalen die größte Anzahl Onlineshops registriert.

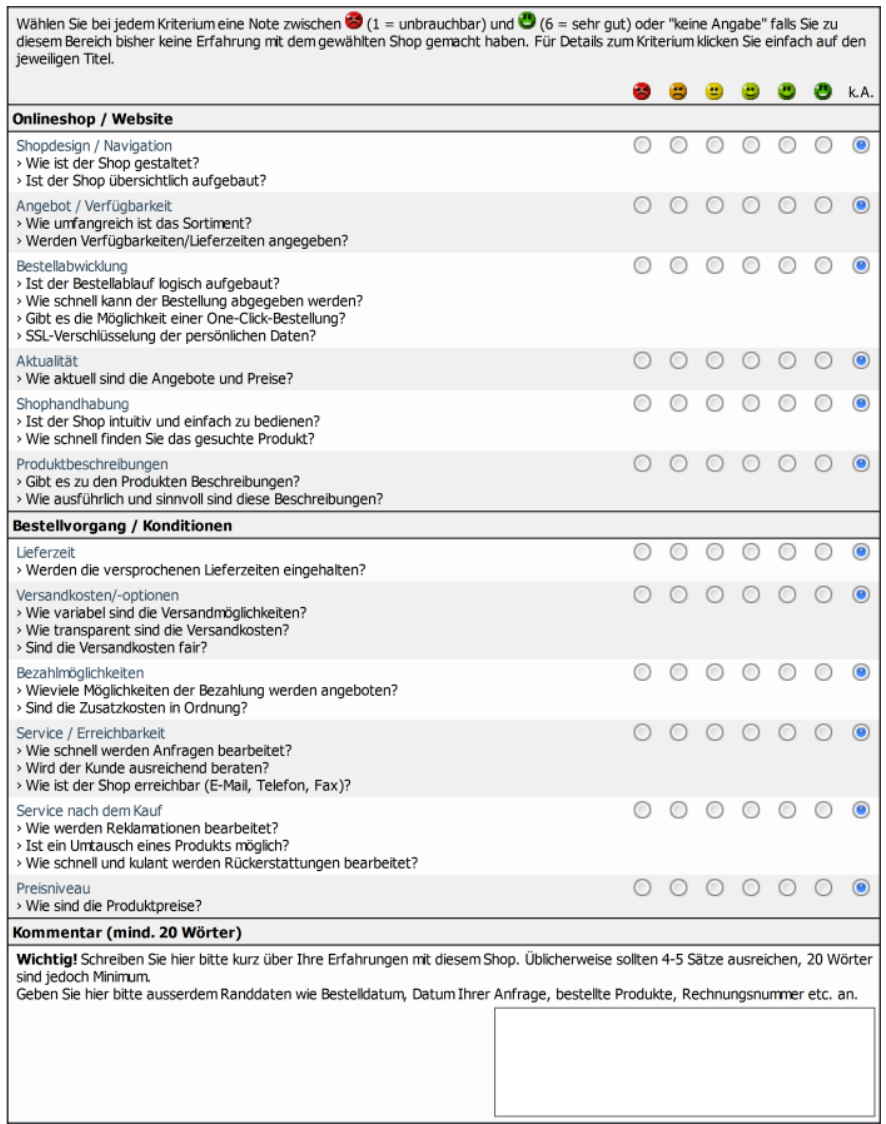

Abbildung 1: Bewertungsraster von Onlineshops bei www.toppreise.ch

\subsection{Extraktion und Aufbereitung der Daten}

Die Informationen bei toppreise.ch wurden, wie in Abbildung 2 gezeigt, schrittweise extrahiert und aufbereitet. Sie werden im Portal in semistrukturierter Form angeboten (Abiteboul et al. 1999, S. 11) . Das ermöglichte den Autoren, die Informationen auszulesen und in mehreren Schritten in eine Tabelle überzuführen, die für eine statistische Auswertung mit SPSS Statistics benötigt wird.

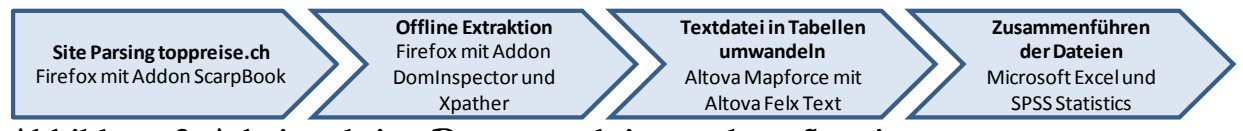

Abbildung 2: Arbeitsschritte Datenextraktion und -aufbereitung 
Die „Händlerliste“ von www.toppreise.ch ${ }^{2}$ mit den 342 verlinkten Händlerdetailseiten wurde mit der aktuellen Version des Webbrowsers „Firefox“3 und dem Add-on „ScrapBook“4 aufgerufen, und auf einem lokalen Datenträger gespeichert. Die auf diese Weise gespeicherten 342 HTML-Seiten wurden im nächsten Schritt über die Firefox Add-ons „DOM Inspector ${ }^{{ }^{\circ 5}}$ und „XPather" ${ }^{\text {"6 } 6}$ einzeln ausgelesen und in eine Textdatei gespeichert. Der Zugriff, auf die in den HTML-Seiten gespeicherten semistrukturierten Informationen, erfolgte über mehrere XPathZuweisungen (Berglund et al. 2007), die mit dem Firefox Add-on „Firebug ${ }^{67}$ erstellt wurden. Im Schritt „Extraktion“ wurden die erstellten Textdateien mit „Altova MapForce“8 und „Altova FlexTex“" in Tabellen umgewandelt. Im letzten Schritt wurden die Tabellen mit „Microsoft Excel“10 und „SPSS Statistics“11 in eine einzige Datendatei zusammengeführt.

\section{Ergebnisse - Explorative Faktorenanalyse}

toppreise.ch gibt für Onlineshops, die mindestens zehn Bewertungen aller 12 Kriterien erhalten haben (siehe Abbildung 1), die Durchschnittsbewertung des jeweiligen Kriteriums aus. 250 der 342 registrierten Onlineshops haben mindestens eine Benutzerbewertung erhalten, wobei $126 \mathrm{mehr}$ als 10 Bewertungen erhalten haben. Diese 126 Onlineshops haben insgesamt 17098 Bewertungen bekommen, die in die Durchschnittsbewertung der Kriterien einfließen.

Zur Untersuchung der Notwendigkeit des Kriterienkatalogs von toppreise.ch mit 12 Bewertungskriterien wird die explorative Faktorenanalyse angewendet. Diese ist ein oft verwendetes Verfahren der multivariaten Statistik, um aus gegebenen Variablen auf zugrundeliegende latente Faktoren zu schließen. Das Verfahren basiert auf der Ermittlung von Korrelationen und Signifikanzen zwischen gegebenen Variablen (Backhaus et al. 2006, S. 259-336). Die Faktorenanalyse ist ein mehrstufiges Verfahren und relativ komplex, da jede Stufe einzeln bewertet werden muss. Damit wird sichergestellt, dass die am Ende extrahierten Faktoren eine entsprechende Aussagekraft besitzen.

Grundsätzlich muss vor der Anwendung der explorativen Faktorenanalyse das „Subjekte zu Variablen-Verhältnis“ geprüft werden. Das Verhältnis von 126 Onli-

\footnotetext{
2 http://www.toppreise.ch/index.php?p=15, Zugriff am 1. August 2009

3 http://www.mozilla-europe.org/de/firefox, Zugriff am 11. Juli 2009

${ }^{4}$ https://addons.mozilla.org/de/firefox/addon/427, Zugriff am 15. Juli 2009

${ }^{5}$ https://addons.mozilla.org/de/firefox/addon/6622, Zugriff am 20. Juli 2009

${ }^{6}$ https://addons.mozilla.org/de/firefox/addon/1192, Zugriff am 20. Juli 2009

7 https://addons.mozilla.org/de/firefox/addon/1843, Zugriff am 20. Juli 2009

8 http://www.altova.com/de/mapforce.html, Zugriff am 1. September 2009

${ }^{9}$ http://www.altova.com/de/mapforce/text-konvertieren.html, Zugriff am 1. September 2009

$10 \mathrm{http}: / /$ office.microsoft.com/de-at/excel/FX100487621031.aspx, Zugriff am 1. September 2009

11 http://www.spss.com/statistics, Zugriff am 1. September 2009
} 
neshops (Subjekte) mit 12 Kriterien (Variablen) entspricht 10:1. Dies ist gemäß Nunnally (1978, S. 421) das mindest geforderte Verhältnis für die Durchführung einer explorative Faktorenanalyse.

\subsection{Extraktion der Faktoren}

In der vorliegenden Analyse werden mit SPSS die Faktoren über die HauptachsenFaktorenanalyse extrahiert und für die Zuordnung der Variablen zu den Faktoren mit der Varimax-Methode rotiert. Diese Parameter sind die gängigsten, da sie zuverlässige und gut interpretierbare Ergebnisse liefern (Backhaus, Erichson et al. 2006, S. 259-336).

Um starke, hinter den oben aufgeführten Bewertungskriterien stehende Faktoren zu erhalten, werden mehrere Faktorenanalysen durchgeführt. Schrittweise werden einzelne Variablen (Kriterien) mit zu niedriger Ladung oder mit einer Kreuzladung auf die Faktoren aus der nächsten Analyse entfernt (Costello und Osborne 2005, S. 3).

Für die Extraktion der Faktoren werden zu Beginn alle zwölf bei toppreise.ch vorhandenen Bewertungskriterien als Variablen gewählt (siehe Abbildung 1). Aus der abschließenden Faktorenanalyse werden aus den oben genannten Gründen die folgenden drei Variablen entfernt:

- Angebot / Verfügbarkeit

- Bezahlmöglichkeiten

- Preisniveau

Anhand der übrigen Variablen wurden zwei Faktoren über den Eigenwert (siehe Tabelle 2) wie über die Analyse des Screeplot extrahiert (siehe Abbildung 3).

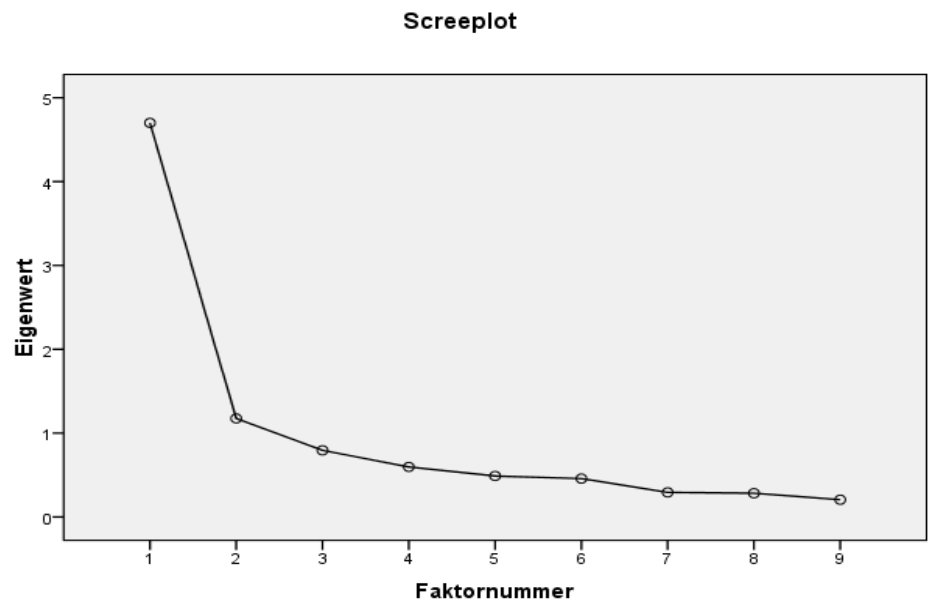

Abbildung 3: Screeplot zur Faktorenanalyse 
Das KMO-Kriterium (Kaiser und Rice 1974) dieser Analyse beträgt bei 0.859 . Dieser Wert wird als „,meritorius“ (,verdienstvoll“) bezeichnet und stellt somit einen sehr guten Wert für die gewählte Stichprobe dar.

Tabelle 2: Resultat der Faktorenanalyse

Cronbach's $\alpha /$ Eigenwert

Faktorladung

$\alpha=0.883$

Faktor 1: Auftragsabwicklung / Serviceleistung

Eigenwert $=4.700$

Lieferzeit

.830

Service - Erreichbarkeit

.774

Bestellabwicklung

.760

Service nach dem Kauf

.637

Aktualität

.608

Versandkosten od. -optionen

.520

$$
\alpha=0.792
$$

Faktor 2: Bedienbarkeit / Informationsdarstellung

Eigenwert $=1.175$

Shopdesign - Navigation

.928

Shophandhabung

.672

Produktbeschreibungen

Extraktionsmethode: Hauptachsen-Faktorenanalyse.

Rotationsmethode: Varimax mit Kaiser-Normalisierung.

Die Cronbach-Alpha-Werte für beide extrahierten Faktoren von je über 0.70 (Nunnally 1978, S. 245) deuteten zudem auf eine stabile und konsistente Stichprobe hin (siehe

Tabelle 2).

\subsection{Interpretation der Faktoren}

Dieses, zunächst rein syntaktische, Ergebnis muss in einem weiteren Schritt interpretiert und auf logische Zusammenhänge hin überprüft werden. Dies ist notwendig, um eine Aussage hinsichtlich der abstrakten Bedeutung der Faktoren machen zu können. Die beiden hinter den gewählten Variablen stehenden Faktoren können wie folgt interpretiert werden (siehe

Tabelle 2):

Faktor 1: Auftragsabwicklung / Serviceleistung: Die Variablen, die diesem Faktor zugeordnet werden, beziehen sich alle auf Bewertungskriterien, die entweder mit der Auftragsabwicklung des Onlineshopbetreibers oder mit der Serviceleistung zu tun haben. Wobei die Auftragsabwicklung als eine Serviceleistung eines Anbieters gesehen werden kann. 
Faktor 2: Bedienbarkeit / Informationsdarstellung: Die Variablen hinter diesem Faktor stehen alle für Bewertungskriterien, die entweder die Handhabung des Onlineshops betreffen oder mit der im Onlineshop angebotenen Information zu tun haben. Die Handhabung eines Onlineshops wird meistens durch eine übersichtliche Darstellung der Informationen unterstützt.

Entgegen der ursprünglichen Eingliederung in zwei Bereiche durch den Portalbetreiber (Onlineshop / Website und Bestellvorgang / Konditionen) ergeben sich zwei neue Faktoren, wobei beispielsweise im Faktor 1: Auftragsabwicklung / Serviceleistung Kriterien aus beiden ursprünglichen Bereichen zu finden sind.

Nach der vorliegenden Analyse besteht die Möglichkeit, die 12 Kriterien auf fünf zu reduzieren und die zwei Bereiche aufzuheben:

- Angebot / Verfügbarkeit

- Bezahlmöglichkeiten

- Preisniveau

- Auftragsabwicklung / Serviceleistung

- Bedienbarkeit / Informationsdarstellung

Allerdings sollte dies durch weitere empirische Beobachtung begleitet werden.

\section{Diskussion}

Den richtigen Kriterienkatalog für Bewertungen von Inhalten im Internet festzulegen ist keine triviale Aufgabe. Hierbei kann es unterschiedliche Sichtweisen geben. Zum einen kann eine einfache Aussage, wie beispielsweise bei eBay, effizient einen generellen Überblick über das Transaktionsverhalten der Partner geben. Zum anderen können ausführliche Bewertungen wie bei holidaycheck.de einen guten Überblick über eine Reihe von Eigenschaften eines Produktes geben.

In diesem Papier wurde aufgezeigt, dass ein Teil der Bewertungskriterien letztlich die gleiche Aussage treffen können. Der Kriterienkatalog differenziert die Onlineshops in vielen Punkten nicht. D.h. es ist nicht klar, ob die unterschiedlichen Bewertungen innerhalb der Kriterien zufällig oder systematischer Natur sind, bzw. der Unterschied der Bewertungen schlicht zu gering sind. Gleichzeitig wurde eine Methode aufgezeigt, wie Betreiber von Bewertungsportalen die Kriterien auf Redundanzen hin überprüfen können. Ziel sollte es in der Regel sein, mit möglichst wenigen Kriterien bestmöglich die Inhalte differenzieren zu können, um eine möglichst breite Basis an Nutzern zu erreichen, die eine Bewertung abgeben. Dies wird mit einem umfangreichen - ggf. auch redundanten - Kriterienkatalog nicht unbedingt positiv beeinflusst. Wichtig ist bei diesem Ansatz aber, dass bei einer Reduktion des Kriterienkatalogs, diese immer durch weitere empirische Beobachtungen begleitet werden muss, um sicherzustellen, dass die Interpretation des entstandenen Faktors korrekt ist.

Selbstverständlich können Kriterien vereinzelt auch als Prüfgrößen angedacht bzw. in den Kriterienkatalog eingebaut werden, um die Plausibilität von Bewertun- 
gen untermauern zu können. Auch für die Entwicklung von Prüfkriterien kann man die vorgestellte Methodik der Analyse einsetzen. Sie eignet sich um die Validität des Konstrukts zu untermauern. Es gibt mannigfaltige Einsatzmöglichkeiten der Faktorenanalyse im Bereich von Bewertungsportalen. Sie helfen, diese Portale zu verbessern.

Der in diesem Papier beschriebene methodische Ansatz zur Identifikation von differenzierenden Bewertungskriterien wird demnächst im Rahmen weiterer Studien auf andere Bewertungsplattformen wie hrs.de, holidaycheck.de usw. angewendet.

\section{Literatur}

Abiteboul S, Buneman P, Suciu D (1999) Data on the Web: From Relations to Semistructured Data and XML. Morgan Kaufmann, San Francisco.

Backhaus K, Erichson B, Plinke W (2006) Multivariate Analysemethoden. Eine anwendungsorientierte Einführung. Springer, Berlin.

Berglund A, Boag S, Chamberlin D, Fernández MF, Kay M, Robie J, Siméon J (2007) XML Path Language (XPath) 2.0. http://www.w3.org/TR/xpath20/. Abruf am: 2009-08-17.

Bryan D, Lucking-Reiley D, Prasad N, Reeves D (2000). Pennies from eBay: The Determinants of Price in Online Auctions, Department of Economics, Vanderbilt University.

Cabral LMB, Hortaçsu A (2006) The Dynamics of Seller Reputation: Theory and Evidence from eBay.

http://pages.stern.nyu.edu/ lcabral/workingpapers/CabralHortacsu_Mar06.p df. Abruf am: 2007-04-10.

Costello AB, Osborne JW (2005) Best Practices in Exploratory Factor Analysis: Four Recommendations for Getting the Most From Your Analysis. Practical Assessment, Research \& Evaluation. 10(7): 9.

Dellarocas C (2000) Immunizing Online Reputation Systems Against Unfair Ratings and Discriminatory Behaviour. In: Jhingran A (Hrsg) ACM Conference on Electronic Commerce (EC-00), Minneapolis, Minnesota, USA: 8.

Dellarocas C (2003) The Digitization of Word of Mouth: Promise and Challenges of Online Feedback Mechanisms. Management Science 49(10): 1407-1424.

Friedman EJ, Resnick P (1999) The Social Cost of Cheap Pseudonyms. http://www.si.umich.edu/ presnick/papers/identifiers/081199.pdf. Abruf am: 2007-04-10. 
Hage S (2006) Tim O'Reilly - Der Web-Meister. http://www.managermagazin.de/it/artikel/0,2828,449911,00.html. Abruf am: 2009-07-12.

Kaiser HF, Rice J (1974) Little Jiffy, Mark IV. Journal of Educational and Psychological Measurement 34: 111-117.

Kollock P (1999) The Production of Trust in Online Markets. Advances in Group Processes 16(1): 16.

Melnik MI, Alm J (2002) Does a Seller's eCommerce Reputation Matter? Evidence from eBay Auctions. Journal of Industrial Economics 50(3): 337-349.

Nunnally JC (1978) Psychometric Theory. Mcgraw-Hill College.

Resnick P, Zeckhauser R, Friedman E, Kuwabara K (2000) Reputation Systems. Communications of the ACM 43(12): 45-48.

Riemer K, Korn D (2007) Design von Reputationssystemen in Online-Auktionen Eine vergleichende Marktanalyse. In: Oberwies A, Weinhardt C, Gimpel H, Koschmider A, Pankratius V, Schnizler B (Hrsg) 8. Internationale Tagung Wirtschaftsinformatik - eOrganisation: Service-, Prozess-, MarketingEngenieering, Karlsruhe: 851-868.

Shmatikov V, Talcott C (2005) Reputation - based trust management. Journal of Computer Security 13(1): 167-190.

van der Heijden H (2004) User Acceptance of Hedonic Information Systems. MIS Quarterly 28(4): 695-704. 